UC. 20

Reporting Date: July 1974 Issued: August 1974

\title{
Magnetically Protected First Wall for a Laser-Induced Thermonuclear Reaction
}

by

Joseph J. Devaney 
This report was prepared as an account of work sponsored by the United States Government. Neither the United States nor the United States Atomic Energy Commission, nor any of their employees, nor any of their contractors, subcontractors, or their employees, makes any warranty, express or implied, or assumes any legal liability or responsibility for the accuracy. completeness or usefulness of any information, apparatus, product or process disclosed, or represents that its use would not infringe privately owned rights.

In the intisest of prompt distribution, this LAMS report was not edited by the Technicai Information staff.

Printed in the United States of America. Available from

National Technical Information Service

U.S. Department of Commerce

5285 Port Royal Road

Springfield, Virginia 22151

Price: Printed Copy \$4.00 Microtiche \$1.45 
MAGNETICALLY PROTECTED FIRST WALL FOR A LASER-INDUCED THERMONUCLEAR REACTOR

by

Joseph J. Devanty

ABSTRACT

A modest magnetic fleld $(3-k G)$ is found to protect a cylindrical wall (240-cm radius) against both the alpha pulse and the DT debris plasma from a laser induced thermonuclear microexplosion (100-MJ). our calculations indicace that the Debye lengths are sufficiently small compared to our minimum possible dimensions so that both the $\alpha$ particles and the debris behave collectively. Our expiosion compresses the magnetic field to as much as $21 \mathrm{kG}$ against a regligiblcresistance coil or cylindrical sleeve. Expansions from 102 to $222 \mathrm{~cm}$ are posstble depending on the residual gas density. The expanding plasmas are demonstrated to be weakly stable over a large pat $t$ of their expansion. The instabilities that do develop are slow: allowing time for adequate pressure relief at the ends.

I. GENERAL̈

This report gives the reasoning and calculations supporting use of simple magnetic fields to protect the first or inner wall of the reaction cavity against the products of a reference laser-impioded DT pellet of 100-MJ yield. Briefly, the reason that simple fleld geometries are adequate for laser-induced controlled thermonuclear reactions, LCTR, but not for ordinary controlled thermonuclear reactions, CTR, (Sherwood) lies in the shorter plasma time of confinement needed. In fact for LCTR the magnetic field need not even confine the exploding plasma, but need only decelerate it sufficlently to prevent wall damage. However, our preliminary calculations here indicate actual cylindrical confinement, thus protecting the first wall for times beyond a plasma recoll back toward the axis.

Our geometry $i$; the simplest, a microexplosion occurring on the axis of a long solenoid, see Fig. 1. Our objective is to protect the wall of the solenoid, but yet allow the plasma to stream toward the ends.

As in all magnetic confinement, the critical question is whether instability permits energetic plasma penetration to the wall, in this case before

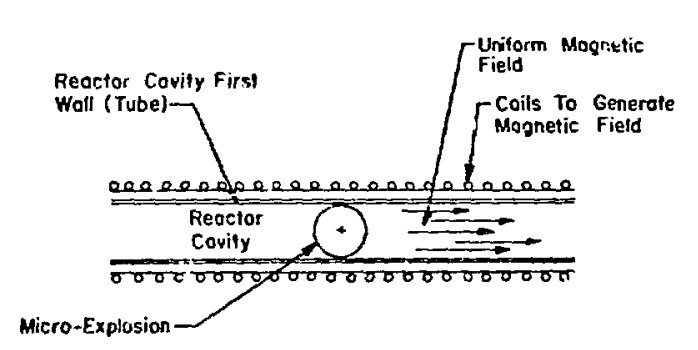

Fig. 1. Magnetically protected cavity wall.

the plasma rebounds from the compressed magnetic field. The answer for our reference design is negative--a conculstion we will support with the following three indicative calculations:

1. Calculation of flate instabilities ${ }^{1,2}$ at pressure equilibrium by a method suggested by W. Riesenfeld. (The author is grateful to Dr. Riesenfeld for this "rule of thumb" and his continued advice.)

2. Flute instability criteria for an expanding superconducting plasma shell into an ambient uniform vacuum magnetic fleld. ${ }^{3}$ 
3. Differential Larmor radius stabilization of otherwise weakly unstable confined plasmas. ${ }^{4}$ Reference Intitial Conditions 5,6

We postulate $2.2 \times 10^{19}$ outwardly directed $\alpha$ particles with an energy of $2 \mathrm{MeV}$, beginning in a shell of $0.0116-\mathrm{cm}$ thickness at a radius of $0.13 \mathrm{~cm}$ for a total energy of $7 \mathrm{MJ}$. In addition, a fully ionized plasma of 50:50 DT mixture expands in (roughly) a spherical she11 of $0.0116 \mathrm{~cm}$ wich an Initial outer radius of $0.13 \mathrm{~cm}$, an initial momentum of $1.39 \times 10^{6} \mathrm{~g} \cdot \mathrm{cm} / \mathrm{s}$ with a total energy of $15 \mathrm{MJ}$. Expansion in a shell is also a worst-case test of wall prorection, because the magnetic fleld must then contain the highest pressure. These plasmas originate simultaneously on the axis of a solenoid of negligible resistance, of radius $240 \mathrm{~cm}$, producing a uniform steady cylindrical field of $3 \mathrm{kG}$. The solenoid contains residual 50:50 DT gas of density rangling from 0 to $4.15 \times 10^{-7} \mathrm{~g} / \mathrm{cm}^{3}$.

\section{COLLECTIVE PLASMA BEHAVIOR}

A whole plasma behaves collectively if its minimum dimension exceeds the Debye length, $\lambda_{D}, 7$ defined by

$$
\lambda_{\mathrm{D}}=\sqrt{\mathrm{kT} / 4 \pi \mathrm{Ne}^{2}}
$$

where $k$ is Boltzman's Constant, $T$ is the absolute temperature, $\mathrm{N}$ is the electron density, and $\mathrm{e}$ is the charge.

For a worst-case test of the $\alpha$ particles we take $\mathrm{kT}=2 \mathrm{MeV}$ and confine them to a shell of 0.116 mm thickness giving $\lambda_{\mathrm{D}}<6 \times 10^{-7} \mathrm{~cm}$ which is much less than the shell thickness $0.0116 \mathrm{~cm}$ at an outer radius $R=0.0116 \mathrm{~cm}$. If we expand the alphas adiabatically as an ideal gas unt 11 stagnation at a radius of $209 \mathrm{~cm}$, there $\lambda_{0}<2 \times 10^{-5} \mathrm{~cm}$, which is also much less than $0.0116 \mathrm{~cm}$. Similarly, for a worst sase, if we expand 1sothermally to $209 \mathrm{~cm}$ and take $\mathrm{kT}=2 \mathrm{MeV}$ we find $\lambda_{\mathrm{D}}<0.0178 \mathrm{~cm}$, which is of the order of $0.0116 \mathrm{~cm}$, so that we can treat the reference $\alpha$-particle burst as a directed plasma working against the magnetic field.

The Larmor radius of a $2-\mathrm{MeV} \alpha$ particle in a 3-kG field is $135.8 \mathrm{~cm}$. The electron Larmor radius is $\sim 1 / 80 \mathrm{C} 0 \mathrm{th}$ of that, but separation of $2.2 \times 10^{19}$ 2-MeV alphas from thelr electrons would give rise to a potential of $\sim 5 \times 10^{10} \mathrm{v}$, so separation cannot occur and ambipolar or collective behavior is supported.
The DT debris remains a collective plasma because the Debye length, $\lambda_{D}$, vartes from $\sim 3 \times 10^{-9}$ to $10^{-7} \mathrm{~cm}$ from a radius of $0.013 \mathrm{~cm}$ to a radius of $210 \mathrm{~cm}$, respectively.

\section{MAGNETIC PROTECTION}

In keeping with the philosophy of worst-case investigation, we calculate the equatorfal containment of the plasma by applying equatorial parameters to spherical geometry. In our case, the actual geometry is cylindrical, which will relieve pressure axla11y, thus imposing a lesser containuent burden than indicated from our spherical-geometry calculation.

Because we assume that solenoid resistance is negligible and that the coil windings are arbitrarily tight (or else use an internal conducting cylindricial sleeve), a compressed magnetic field cannot penetrate the coil windings; we may, by conservation of flux, calculate the compressed fieid, $B$, to be that of the uncompressed field, $B_{0}$, times the inverse rat to of cross-sectional areas, thus:

$$
B=B_{0} /\left[1-\left(R / R_{0}\right)^{2}\right],
$$

wich gives rise to a magnetic pressure, $p_{m}$, resisting plasma expansion:

$$
P_{m}=\frac{B^{2}}{8 \pi} \text { (Gaussian unlts). }
$$

The work done by the expanding plasma, $W$, is that done on the magnetic field, $W_{m}$, plus that compressing the residual gas, $w_{\text {gas }}$. The pressure of residual gas we take to be a power law in the Taylor-Sedov region, 6

$$
P_{\text {gas }}=4.4 \times 10^{13} \mathrm{R}^{-3} \text { (cgs units), }
$$

leading to

$$
\begin{gathered}
W_{g}=\int_{R_{1}}^{R} P_{g} d V=\int_{R_{1}}^{R} 4.4 \times 10^{13} R^{-3} 4 \pi R^{2} d R \\
=5.53 \times 10^{14} \text { ln }\left|\frac{R}{R_{1}}\right| .
\end{gathered}
$$

At less than $R_{1}$ where Taylor-Sedov theory breaks down we assume that $P_{\text {gas }}$ is roughly constant, ${ }^{8}$ so in that range,

$$
W_{g}=P_{g} \frac{4 \pi}{3} R^{3}
$$


The magnet1c work 13

$$
\begin{aligned}
& W_{m}=\int P_{m} d V=\int_{0}^{R} \frac{B_{o}^{2}}{8 \pi\left[1-\left(\frac{R}{R_{o}}\right)^{2}\right]} 4 \pi R^{2} d R \\
& W_{m}=\frac{{ }_{0}^{B}{ }^{2} R_{0}^{3}}{4}\left[\frac{R R_{0}}{R_{0}{ }^{2}-R^{2}}-\frac{1}{2} \ln \left|\frac{R_{0}+R}{R_{0}-R}\right|\right]
\end{aligned}
$$

where $R_{0}$ is the coll radius and $B_{o}$ is the intelal uniform field.

The range of cases important to LCTR is spanned by :

1. Zero-density residual gas, $\rho_{0}=0$, at the time of explosion, learing the magnetic field as the only restraining force;

2. Low-density residual gas, $10^{12}$ DT particles/ca ${ }^{3}$;

3. High (relative) density residual gas, $10^{17}$ DT particles $/ \mathrm{cm}^{3}$. Higher densities place unacceptable constraints on the admission of laser light onto the pellet through the chamber atmosphere.

By equating the directed energy of 2-MeV alphas and the total energy (15-MJ) in the debris plasma to the work done in expansion we can calculate the maximum equatorial expansion of the alphas and of the plasma.

Then, for Case 1, $\left(\rho_{0}=0\right)$, the 2-MeV alpha pulse will be reversed at a radius of $209 \mathrm{~cm}$ giving a compressed field of $12.4 \mathrm{kG}$. The plasma pulse will be reversed at less than $222 \mathrm{~cm}$ for a maximum fleld of less than $20.9 \mathrm{kG}$.

For Case 2, $\left(\rho_{0}=4.15 \times 10^{-12} \mathrm{~g} / \mathrm{cm}^{3} \mathrm{DT}\right)$, the plasma expands to less than $222 \mathrm{~cm}$ with a maximum fleld less than $20.4 \mathrm{~kg}$.

Case $3\left(\rho_{0}=4.15 \times 10^{-7} \mathrm{~g} / \mathrm{cm}^{3} \mathrm{DT}\right)$, the alpha pulse expands to $208-\mathrm{cm}$ maximum radius with a corresponding maximum fleld of $11.9 \mathrm{~kg}$. These values are 11ttle different from those for vacuum. We used an average stopping power in DT of $1.6 \times 10^{3} \mathrm{MeV}-\mathrm{cm}^{2} / \mathrm{g}$ for the $\alpha$ particles. On the other hand, the original explosion debris plasma expands to less than $14.8 \mathrm{~cm}$. The original plasma is thus easily decelerated by the residual gas. of course, the residual gas is itself shocked and fonized. The total excursion of tonlzed material therefore exceeds $14.8 \mathrm{~cm}$ and must be otherwise determined, which we do from momentum considerations.
The residual gas can decelerate but not subtract momentum from the explosion; momentum can be subtracted only by the magretic ficld (or the wall).

If such outward momentum is $p$, then

$$
\mathrm{p}=\int_{0}^{\mathrm{X}} F(R) \frac{d t}{d R} d R
$$

where $\mathrm{R}$ is the radius at which the shocked ionized gas is brought to rest and $P(R)$ is the decelerating force. For simpiicity we take all impacted gas to be Ionized. However, in fact the magnetic field cannot protect the first wall against a pressure pulse of unionfzed residual gas. Should the latter be strong, one must also mechanically strengthen the first wall.

Conservation of momentum gives $\mathrm{dR} / \mathrm{dt}$ as a function of $R$ :

$$
p=m v=(m+d m)(v+d v)+v d m+m d v=0 .
$$

Solving, the velocity, $v$, and moving mass, $m$, are related by

$$
v=\frac{c}{m}
$$

where $c$ is a constant.

Thus,

$$
v=\frac{d R}{d t}=\frac{c}{m_{0}+\rho_{0}\left(4 \pi R^{3} / 3\right)} .
$$

Initially, $v=1.07 \times 10^{8} \mathrm{~cm} / \mathrm{s}$ if the effective kinet de energy is half the total energy; ${ }^{8}$ and $v=1.97 \times 10^{8} \mathrm{~cm} / \mathrm{s}$ if all the energy is kinetic. For our specific initial conditions the constant, $c$, equals $1.4 \times 10^{6}$ to $2 \times 10^{6} \mathrm{~g} \mathrm{~cm} / \mathrm{s}$.

Our restraining force here is wholly magnetic:

$$
F(R)=P_{m} A=\frac{B_{0}^{2}}{8 \pi\left[1-\left(\frac{R}{R_{0}}\right)^{2}\right]^{2}} \cdot 4 \pi R^{2} .
$$

The integral for the momentum, p, becotaes:

$$
P=\frac{B_{0}^{2} R_{0}^{4}}{2 c} \int_{0}^{R} \frac{m_{0}+\rho_{0}(4 \pi / 3) R^{3}}{\left(R_{0}^{2}-R^{2}\right)^{2}} R^{2} d R
$$




$$
\begin{aligned}
& \mathrm{p}=\frac{\mathrm{B}_{\mathrm{O}}{ }^{2} \mathrm{R}_{\mathrm{O}}^{2}}{2 \mathrm{c}}\left(\frac{\mathrm{m}_{\mathrm{O}}}{2} \cdot \frac{\mathrm{R}}{\left[I-\left(\mathrm{R} / \mathrm{R}_{\mathrm{O}}\right)^{2}\right)}-\frac{\mathrm{R}_{\mathrm{O}}}{2} \ln \left|\frac{1+\left(R / \mathrm{R}_{\mathrm{O}}\right)}{1-\left(R / \mathrm{R}_{\mathrm{O}}\right)}\right|\right) \\
& +\frac{a}{2} R_{0}^{4}\left\{-\left[1-\left(R / R_{0}\right)^{2}\right]+\left[1-\left.\left(R / R_{0}\right)^{2}\right|^{-1}\right.\right. \\
& \left.+2 \ln \left|1-\left(R / R_{0}\right)^{2}\right| \mid\right)
\end{aligned}
$$

Substituting our parameters, Case 3, all the (shocked) plasma is brought to rest between $R=102$ $\mathrm{cm}, \mathrm{B}=3650 \mathrm{G}(50 \%$ in kinetic energy $)$ and $R=113$ $\mathrm{cm}, \mathrm{B}=3850 \mathrm{G}$ (al1 in kinetic energy).

\section{PLASMA STABILITY}

\section{Plasma Instability at Pressure Equilibrium}

At pressure equilibrium between the plasma and the magnetic field, that is, at stagnation, we may expect flute irregularities to grow as: ${ }^{1,2}$

$$
\exp (t / \tau)
$$

Where

$$
T \sim 2 \pi R /\left(V_{A} \sqrt{n}\right)
$$

and $v_{A}=B^{2} / 4 \pi \rho$ is the Alfven velocity: $n$ is the number of flutes (asymetric explosions, $n=1$, are taken to be the most probable for LCTR); and $\rho$ is the plasma mass density. As a figure of merit for the stability of expansion against our magnetic field we calculate the time constant, $\tau$, times the effective axial velucity, $v_{a}$. This value, a distance, call it $D_{a}$, is a conservative stinate of the axial expansion that may occur befo $e$ the onset of serlous instabilities. The estimate is conservative because it is made at highest pressure (equilibrium, $B=1$ ) which occurs only momentarily. We may expect, as we show later, less instability, even stability at $B<1$.

Case 1, no residual gas - The alphas treated as a plasma have a time constant $\tau=5.7 \times 10^{-5} \mathrm{~s}$, with a velocity of $9.8 \times 10^{8} \mathrm{~cm} / \mathrm{s}$, giviig $\mathrm{D}_{\mathrm{a}}$ $34000 \mathrm{~cm}$. The plasma has a tline constant $\tau=3.2$ $x 10^{-4} \mathrm{~s}$; taking a velocity of $10^{8} \mathrm{~cm} / \mathrm{s}, 6$ we get $D_{a} \sim 56000 \mathrm{~cm}$.

Case 2, residual DT gas;density, $10^{12}$ particles/ $\mathrm{cm}^{3}$ - The plasma has an equilibrium flute-instability time constant of $\tau=3 \times 10^{-4} \mathrm{~s}$ which, Integrating the velocity profile of Ref. 8, gives $D_{a} \sim 4600 \mathrm{cs}$.
Cass: 3, residual DT gas; density, $10^{17}$ part 1cles $/ \mathrm{cm}^{3}$ - The alpha plasma has a time constant $\tau=5.9 \times 10^{-5} \mathrm{~s}$, which is long enough for the axially direcied alphas to be stopped in the gas at $D_{a} \simeq 3000 \mathrm{~cm}$. We used an average stopping power of $1.6 \times 10^{3} \mathrm{MeV}-\mathrm{cm}^{2} / \mathrm{g}$ for alphas in DT, treating the alphas in this regime as particles, not as gas. The plasma has a time constant $\tau=5.2 \times 10^{-4} \mathrm{~s}$, which allows the shocked plasma to move axially for a distance $\mathrm{D}_{\mathrm{a}} \simeq 600 \mathrm{~cm}$.

In every instance the magnetic field has persisted uniformly enough until the explosive debris is well on its way outward along the axis of the solenold. We now turn to a more complete description of the expansion of a plasma shell into a vacuum magnetic field.

2. Plasma Stabi'ity of a Spherical Shell

Flute-instability growth of an initially

spherical superconducting plasma shell into a large vacuum magnetic field is given by formulae proportional to the growth term $e^{t / \tau}$ where the time constant, $\tau$, is (from Poukey ${ }^{3}$ ):

$$
\begin{gathered}
\tau=(2 / 3)(n \alpha)^{-1 / 2} n \alpha \gg 1 \\
\tau=\left(\frac{32}{81}\right)^{2 / 3}(n \alpha)^{-2 / 3} n \alpha \ll 1,
\end{gathered}
$$

$n$ being the number of flutes and

$$
\alpha \equiv \frac{{ }_{0}{ }_{0}^{2} R_{O}^{3}}{2 M V_{0}^{2}}=\frac{{ }_{{ }_{0}}{ }^{2} R_{0}{ }^{3}}{4 E_{0}} \text { (Gausstan units). }
$$

Here $B_{o}$ is the initial magnetic field, uniform throughout space; $R_{0}$ is the radius of the sphere at $t=0$ expanding outward with an initial velocity $v_{0}$, a total mass $M$, and a total inftial kinetic energy $\mathrm{E}_{0}$. As before we take $\mathrm{n}=1$, since the asymmetry of implosion is most likely a simple off-center ( $n=1)$ type.

For a worst-case calculation we take $E_{\text {as }}$ anly half the cotal plasma energy, $E_{0}=7.5 \times 10^{13} \mathrm{erg}$, and we take $B_{0}$ to be the largest of all cases, $B_{0}=21 \mathrm{kG}$; we then get, for both the alphas and the plasma:

$$
n \alpha-3.4 \times 10^{-10} \ll 1,
$$

and the second time-constant formula ylelds:

$$
\tau-2.4 \times 10^{5} \mathrm{~s},
$$


adequately long indeed for all gases to exit any reasonably sized chamber.

This calculation should be a lower bound ficr instabflity time constants of spherical sinell expansions beginning at a magnet1c field of $3 \mathrm{kG}$ and compressing to a pressure equilibrium of 6 to $21 \mathrm{kG}$. Poukey ${ }^{3}$ did not study expansions into non-vacuurs.

\section{Finite Larmor Radius Stabilization}

Because the Larmor radii of lons and electrons are finite and different, otherwise weakly unstable confined plasmas actually are stable. ${ }^{4}$ The different election and fon Larnor radii may build up a charge separation out of phase with particle drift separation. Because the latter drives the flute instability, the result can be stable oscillation if :

$$
\left(k a_{i}\right)^{2}>\omega_{H} / \Omega_{i},
$$

where $k$ is the wave number, which we have taken as $n / R$, with $n$ being the number of flutes; $a_{i}$ is the ion Larmor radius (gyromagnet $i c$ ), $a_{i}=m_{1} v_{1} c / e_{1} B$; $\Omega_{1}$ is the ion Larmor angular frequency (cyclotron frequency), $\Omega_{1}=e_{1} B / m_{1} c$; and $w_{H}$ is the hydrodynamic growth rate (Taylor instabllity).

The growth rate for Taylor instability under gravity is: ${ }^{9}$

$$
u_{H}^{2}=k g \frac{\rho_{2}-\rho_{1}}{\rho_{2}+\rho_{1}}
$$

for two fluids of density $p_{1}$ and $r_{2}$, $k$ here is the wave number of the Instability, $g$ is the gravitarional acceleration.

A magnetic field behaves as $\rho_{1} \equiv 0$, so $\omega_{\mathrm{H}}{ }^{2}$ $=\mathrm{kg}$ and $\mathrm{g}+\left(\vec{R} / \mathrm{R}^{2}\right)\left(\left.\mathrm{v}_{\|}\right|^{2}+\frac{1}{2} \mathrm{v}_{\perp}{ }^{2}\right)$ for equflibrium. 10 Because $R$ is the radius of curvature of the $B-f$ ield, this radius is identical to our $R ; V_{\|}, V_{1}$ are the velocities parallel and perpendicular to the surface. Because our fluid is not in equiliorium, we must add $\ddot{R} ;$ also, we are confining our study to the equatorial tegion where $V_{||} \equiv 0$, thus $g \rightarrow \ddot{R}+\left(\tilde{R}^{2} / 2 R\right)$.

Using the instantaneous total energy $E=\frac{1}{2} M R^{2}$,

$$
\begin{aligned}
g & =\ddot{R}+E / M R ; \text { hence, } \\
\omega_{H} & =\sqrt{k[\ddot{R}+(E / M R)]} .
\end{aligned}
$$

By substituting Eq. (21) Into Eq. (18) our stability criterion reduces to:

$$
(n / R)^{3 / 2} \frac{2 E_{i} c}{e_{i} B}>\sqrt{\ddot{R}+(E / M R)}=\sqrt{\ddot{R}+\left(\dot{R}^{2} / 2 R\right)}
$$

where $E_{i}$ is the individual ion energy. When this inequality holds we may expect flute stabilization.

For our parameters we find for all cases that the alpha plasma fulfills the inequality to more than 120-cm radius, and the debris plasma to within $3 \mathrm{~cm}$ of the curnaround radius.

We conclude then, by two separate lines of reasoning (Numbers $1+3$ and 2 , of pp, 1,2 and pp. 4,5) that explostons into a magnetic field are either stable or slowly growing unstable. Based on our parameters for a single explosion, $t^{2}$ se stability calculations substantiate beyond re onable doubt chat magnetic wall protection is feasible. For the detailed study of multipl e explosive impacts on stabllity, as in our case where the alpha pulse is followed by the expanding debris, detailed study must be deferred. We do note, however, three reassuring points, namely that:

1. Actual plasma reversal times at maximum radius and magnetic field are short compared to fluteInstability time constants. (For example, in Case 1, the start and return of the plasma to $3 \mathrm{~cm}$ of turnaround radius takes $\sim 6 \times 10^{-8} \mathrm{~s}$ for alpnas versus an alpha instability time constant, $\tau_{\alpha} \sim 6 \times 10^{-5} \mathrm{~s}$. For the explosive debris the 3-cm return time is $-2 \times 10^{-7} \mathrm{~s}$ versus a plasma instability time constant, $\left.\tau_{\text {plasma }}-3 \times 10^{-4} \mathrm{s.}\right)$

2. The alpha-particle flute-instability time constant is sufficiently long for the debris plasma to strike the magnetic field before such growth has gone far, especially for low-density residual gas.

3. Larger pellets, especially those made of higher $Z$ materlals, will attenuate the alpha pulse with the result that our single-pulse calculations become more applicable to the remaining dominant, if not exclusive, debris pulse.

v. CONCLUSTONS

The calculations of this note strongly support the possibility of magnetic protection of a cylindrical cavity wall against energetic alpha particles and plasma debris from microexplosions. Our reference design was based on a $10^{15}$ erg (100-MJ) . T explosion in an inftially uniform 3-kG cylindrical fleld of $240-\mathrm{cm}$ radius. For more details of a 
magnetically protected laser fusion reactor concept see the work of Frank, Freiwald, Merson, and Devaney. 11

\section{ACKNOWLEDGEMENTS}

The early thoughts of this paper were developed during active collaboration on an ANS paper ${ }^{1.1}$ with T. G. Frank, D. Freiwald, and T. Merson. It is a pleasure to acknowledge helpful discussions with W. Riesenfeld and C. M. Gillespie, all of LASL.

\section{REFERENCES}

1. C. L. Longmire, Elementary Plasma Physics, (Interscience, New York, 1967), pp. 241-246.

2. M. N. Rosenbluth and C. L. Longmire, "Stability of Plasmas Confined by Magnetic Flelds," Ann. of Phys. 1,120 (1951).

3a. J. W. Poukey, "Expansion of a Plasma Shell into a Vacuum Magnetlc Field," Phys. Fluids, 12, 1452 (1969).

3b. J. R. Albritton, "A Snowplow Model for the Dynamics of Expanding Plasmas," Yale University Thesis (1973), to be published.
4. M. N. Rosenbluth, N. A. Krall, and N. Rostoker, "Finite Larmor Radius Stabilization of 'Weakly' Unstable Confined Plasmas," Nuclear Fuston Supplement, Pt. 1 (1962), p. 143.

5. T. G. Frank, LASL, private communication (1974).

6. D. Friewald, LASL, unpublished work (1974).

7. D. J. Rose and M. Clark, Plasmas and Controlled Fusion, (MIT Press, Cambridge, MA, 1.969). pp. 161,162 .

8. D. Freiwald, LASL, private commnication (1974).

9. F. H. Harlow and A. A. Ainsden, "Fluld Dynamlcs," Los Alamos Sclentific Laboratory Report LA-4700 (1971).

10. M. N. Rosenbluth and C. Longmire, Ann. of Phys. 1, 120 (1957).

11. T. Frank, D. Freiwald, T. Merson, and J. Devaney, "A Laser Fusion Reactor Concept Utilizing Magnetic Fields for Cavity Wall Protection," presented at First Topical Meeting on the Technology of Controlled Nuclear Fusion, San Diego, California (American Nuclear Society) Apri1 16-18, 1974. 